\title{
TREATMENT OF PAIRING CORRELATIONS IN NUCLEI CLOSE TO DRIP LINES
}

\author{
Nguyen Van Giai ${ }^{\mathrm{a}}$, M. Grasso ${ }^{\mathrm{b}}$, R.J. Liotta ${ }^{\mathrm{c}}$ and N. Sandulescu ${ }^{\mathrm{d}}$ \\ ${ }^{a}$ Institut de Physique Nucléaire, IN2P3-CNRS, \\ Université Paris-Sud, 91406 Orsay Cedex, France \\ b Università di Catania, 95129 Catania, Italy

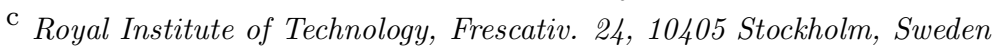 \\ d NIPNE, POB MG-6, Bucharest-Magurele, Romania
}

Keywords: Pairing correlations, HFB, Continuum

\begin{abstract}
We discuss the HFB equations in coordinate representation, a suitable method for handling the full effects of the continuous quasiparticle spectrum. We show how the continuum HFB equations can be solved with the correct asymptotic conditions instead of the discretization conditions which are commonly used in the literature. The continuum HFB method is illustrated with a model where the mean field and pairing field have simple forms. The relationship with the continuum HartreeFock-BCS (HF-BCS) approximation is also discussed. Realistic HFB and HF-BCS calculations based on Skyrme interactions are compared for the case of a neutron-rich nucleus.
\end{abstract}

\section{INTRODUCTION}

In many nuclear systems pairing correlations have an important influence on physical properties. This situation occurs when the energy difference between an occupied orbital and a neighbouring unoccupied one is relatively small, thus enabling a nucleon pair to be promoted to the unoccupied level by the interaction among the pair. Well known theoretical approaches such as the Hartree-Fock-Bogoliubov (HFB) or the Hartree-Fock-BCS (HF-BCS) approximations have been developed [1] to treat the pairing effects in nuclei.

In usual situations, i.e., when the nuclear system is stable and far away from the drip lines the active orbitals are well bound and it may be sufficient to solve the HFB or HF-BCS equations within the discrete 
subspace of those active orbitals. When one approaches a drip line this is no longer true since the active orbitals must also include states belonging to the continuous single-particle spectrum. One of the most convenient tools for the treatment of pairing correlations in the presence of continuum coupling is given by the HFB approach. The standard procedure is to discretize the quasiparticle continuum by solving the HFB equations in a finite basis of orthonormal functions, or by imposing to the solutions of HFB equations, written in coordinate space, a box boundary condition at some distance $R_{b}[2,3,4]$. There may be circumstances where a discretized solution is unsatisfactory unless an extremely large box radius $R_{b}$ is used. Such should be the case for halo nuclei, for instance. In these lectures, we show how the HFB equations can be solved with correct boundary conditions. Based on these solutions we examine how much the continuum, especially the resonant continuum, could affect the pairing properties of nuclei close to the drip lines.

The structure of the lectures is the following: In the next section we introduce the HFB equations in coordinate space $[5,2]$ and in section 3 we examine the solutions which satisfy the correct boundary conditions, i.e., of scattering wave type. In section 4 these considerations are illustrated for the particular case of a square well potential and a constant pairing field of a finite range. Then we discuss how the resonant continuum can be taken into account in HF-BCS approach. Finally we show how the continuum, calculated in different approximations, can affect the pairing properties of a nucleus close to the neutron drip line.

\section{HARTREE-FOCK-BOGOLIUBOV EQUATIONS IN COORDINATE SPACE}

Let us denote by $H$ the hamiltonian of the nuclear system. When treated in the HFB approximation, $H$ will give rise to the mean field and the pairing field in which the quasiparticles are moving. We assume that the two-body effective interactions associated with the Hartree-Fock (HF) mean field and pairing field are zero-range forces so that the total energy $E$ is a functional of the local particle density $\rho(\mathbf{r})$ and pairing density $\kappa(\mathbf{r})$ :

$$
\begin{aligned}
\rho(\mathbf{r}) & \equiv\left\langle H F B\left|\Psi^{+}(\mathbf{r}) \Psi(\mathbf{r})\right| H F B\right\rangle \\
\kappa(\mathbf{r}) & \equiv\left\langle H F B\left|\Psi^{+}(\mathbf{r}) \Psi^{+}(\mathbf{r})\right| H F B\right\rangle
\end{aligned}
$$

where $\Psi^{+}(\mathbf{r})$ is a nucleon creation operator and $|H F B\rangle$ is the HFB ground state. Typically, the self-consistent mean field is generated by a Skyrme-type interaction whereas the pairing field is produced by a zero-range, possibly density-dependent interaction. 
The HFB equations in coordinate space can be expressed in the following form:

$$
\left(\begin{array}{cc}
h-\lambda & \Delta \\
\Delta & -(h-\lambda)
\end{array}\right)\left(\begin{array}{l}
U_{\alpha}(\mathbf{r}) \\
V_{\alpha}(\mathbf{r})
\end{array}\right)=E_{\alpha}\left(\begin{array}{l}
U_{\alpha}(\mathbf{r}) \\
V_{\alpha}(\mathbf{r})
\end{array}\right)
$$

where $\lambda$ is the chemical potential, $h$ is the HF hamiltonian and $\Delta$ is the pairing field. The local nucleon density and pairing density can be written in terms of the solutions $\left(U_{\alpha}, V_{\alpha}\right)$ in the form:

$$
\begin{gathered}
\rho(\mathbf{r})=\sum_{\alpha}\left|V_{\alpha}(\mathbf{r})\right|^{2}, \\
\kappa(\mathbf{r})=\sum_{\alpha} U_{\alpha}(\mathbf{r}) V_{\alpha}^{*}(\mathbf{r}) .
\end{gathered}
$$

For Skyrme forces, the HF and pairing fields may also depend on derivatives of $\rho$ and $\kappa$ and on some local currents because of the velocity dependence of the interaction. Since all densities appearing in the energy functional are local, Eq.(1.2) is a set of coupled differential equations which is highly non-linear because the fields $h$ and $\Delta$ depend themselves on the solutions $U_{\alpha}, V_{\alpha}$ (self-consistency problem). Nevertheless, using Skyrme-type forces brings a major simplification. If one starts instead with a finite range effective force, for instance a Gogny's force, Eq.(1.2) would be a set of coupled integro-differential equations which is usually solved in a harmonic oscillator basis. The general properties of SkyrmeHF hamiltonians are well-known and detailed expressions of $h$ and $\Delta$ for the case of Skyrme forces can be found for instance in Ref. [2].

The Skyrme-HF hamiltonian has the general form:

$$
h=-\nabla \cdot \frac{\hbar^{2}}{2 m^{*}(\mathbf{r})} \nabla+V_{H F}(\mathbf{r}),
$$

where the effective mass $m^{*}$ and HF potential $V_{H F}$ depend on nucleon densities and currents. An important property is that $m^{*}$ tends to the nucleon mass $m$ and $V_{H F}$ tends to zero at infinity, with the same rate as the densities go to zero. We shall use this property in the next section to establish the asymptotic forms of the solutions of Eq.(1.2).

For illustration, we can look at the pairing field calculated with a pairing interaction often used in the literature $[3,4]$ :

$$
V(1,2)=V_{0}\left[1-\left(\frac{\rho\left(\mathbf{r}_{1}\right)}{\rho_{c}}\right)^{\gamma}\right] \delta\left(\mathbf{r}_{1}-\mathbf{r}_{2}\right)
$$


To be meaningful, such an interaction must be used with an energy cutoff in the quasiparticle spectrum, i.e., the summations in Eqs. $(1.3,1.4)$ must be limited to $E_{\alpha} \leq E_{\text {cutoff }}$. In this case, the pairing field is:

$$
\Delta(\mathbf{r})=\frac{1}{2} V_{0}\left[1-\left(\frac{\rho(\mathbf{r})}{\rho_{c}}\right)^{\gamma}\right] \kappa(\mathbf{r}) .
$$

On this example we can see that the local pairing field behaves asymptotically like the local pairing density.

\section{ASYMPTOTIC BEHAVIOUR OF HFB SOLUTIONS}

From the symmetries of Eq.(1.2) it can be seen that, if $\left(E_{\alpha}, U_{\alpha}, V_{\alpha}\right)$ is a solution then $\left(-E_{\alpha}, V_{\alpha}^{*}, U_{\alpha}^{*}\right)$ is also a solution. Thus, we need to consider only one class of solutions and we choose those with $E_{\alpha} \geq 0$.

The asymptotic behaviour of the solutions of Eq.(1.2) has been discussed in detail by Bulgac [5]. Let us consider for simplicity the case of spherical symmetry and write:

$$
\left(\begin{array}{l}
U_{\alpha}(\mathbf{r}) \\
V_{\alpha}(\mathbf{r})
\end{array}\right)=\frac{1}{r}\left(\begin{array}{l}
u_{\alpha}(r) \\
v_{\alpha}(r)
\end{array}\right) \mathbf{Y}_{\alpha}(\hat{r}, \sigma) .
$$

Eq.(1.2) now becomes a one-dimensional equation in the radial coordinate $r$ for each $\alpha=(l, j)$. At very large distances the HF hamiltonian $h$ tends to $-\left(\hbar^{2} / 2 m\right)\left(\frac{1}{r} \frac{d^{2}}{d r^{2}} r-\frac{l(l+1)}{r^{2}}\right)$ (plus a Coulomb potential for protons) while the pairing field $\Delta(r)$ has vanished. The equations for $u_{\alpha}(r)$ and $v_{\alpha}(r)$ are decoupled and one can easily see how the physical solutions must behave at infinity. Thus, for a negative chemical potential $\lambda$, i.e., for a bound system, there are two well separated regions in the quasiparticle spectrum:

- between 0 and $-\lambda$ the quasiparticle spectrum is discrete and both upper and lower components $\left(u_{\alpha}(r), v_{\alpha}(r)\right)$ of the HFB wave function decay exponentially at infinity;

- above $-\lambda$ the quasiparticle spectrum is continuous and the physical solutions are such that at infinity the upper component of the HFB wave function has a scattering wave form (see the next section) while the lower component is exponentially decaying. In what follows the continuous HFB wave functions are normalised to a delta function of energy.

Thus, the summations in Eqs.(1.3-1.4) should in fact include integrations over the continuum of the quasiparticle spectrum:

$$
\rho(\mathbf{r})=\sum_{0 \leq E_{\alpha} \leq-\lambda}\left|V_{\alpha}(\mathbf{r})\right|^{2}+\int_{-\lambda}^{E_{\text {cutoff }}} d E_{\alpha}\left|V_{E_{\alpha}}(\mathbf{r})\right|^{2},
$$




$$
\kappa(\mathbf{r})=\sum_{0 \leq E_{\alpha} \leq-\lambda} U_{\alpha}(\mathbf{r}) V_{\alpha}^{*}(\mathbf{r})+\int_{-\lambda}^{E_{\text {cutoff }}} d E_{\alpha} U_{E_{\alpha}}(\mathbf{r}) V_{E_{\alpha}}^{*}(\mathbf{r}) .
$$

As we have already mentioned in the introduction, most of the practical calculations are done by solving Eq.(1.1) with a box boundary condition $[2,3,4]$, i.e., by requiring that the solutions $U_{\alpha}(\mathbf{r}), V_{\alpha}(\mathbf{r})$ have a node at $r=R$ (they are taken to be identically zero beyond $R$ ). This condition makes the spectrum of $E_{\alpha}$ entirely discrete and allows the use of Eqs.(1.3-1.4) instead of Eq.(1.9). However, it is not clear how accurately one can mock up continuum effects like single-particle resonance contributions by using this discretization procedure. In order to avoid such ambiguities one needs solutions with proper boundary conditions. These solutions are illustrated in the next section for the case of a simple model $[5,6]$.

\section{HFB SOLUTIONS: A SCHEMATIC MODEL}

In what follows we discuss the solutions of HFB equations in coordinate space for a mean field given by a square well potential, of depth $V_{0}$ and radius $a$, and a pairing field, $\Delta$, constant inside the same radius $a$ and zero outside. We suppose also that the Fermi level, $\lambda$, is given. For such a system the radial HFB equations inside the potential well, i.e. for $r \leq a$, are given by:

$$
\begin{aligned}
& \left(\frac{1}{r} \frac{d^{2}}{d r^{2}} r-\frac{l(l+1)}{r^{2}}+\alpha^{2}\right) u_{l j}-\delta^{2} v_{l j}=0, \\
& \left(\frac{1}{r} \frac{d^{2}}{d r^{2}} r-\frac{l(l+1)}{r^{2}}+\beta^{2}\right) v_{l j}-\delta^{2} u_{l j}=0 .
\end{aligned}
$$

where $\alpha^{2}=\frac{2 m}{\hbar^{2}}\left(\lambda+E+U_{0}\right), \beta^{2}=\frac{2 m}{\hbar^{2}}\left(\lambda-E+U_{0}\right), \delta^{2}=\frac{2 m}{\hbar^{2}} \Delta$ and $U_{0}=-\left(V_{0}+V_{s o} \vec{l} \cdot \vec{s}\right)$. The above equations have the following physical solution for any value of the quasiparticle energy :

$$
\begin{aligned}
& u_{l j}=A_{+} j_{l}\left(k_{+} r\right)+A_{-} j_{l}\left(k_{-} r\right), \\
& v_{l j}=A_{+} g_{+} j_{l}\left(k_{+} r\right)+A_{-} g_{-} j_{l}\left(k_{-} r\right),
\end{aligned}
$$

where $j_{l}$ are spherical Bessel functions, $k_{ \pm}=\frac{2 m}{\hbar^{2}}\left(U_{0}+\lambda \pm \sqrt{\left.\left(E^{2}-\Delta^{2}\right)\right)}\right.$ and $\left.g_{ \pm}=\left(E \pm \sqrt{(} E^{2}-\Delta^{2}\right)\right) / \Delta$.

Outside the potential well both $U_{0}$ and $\Delta$ are zero and the HFB equations are decoupled. In this case the type of solutions depends on the quasiparticle energy. Thus, for $E<-\lambda$ the solutions have the form:

$$
\begin{aligned}
& u_{l j}=A h_{l}^{(+)}\left(\alpha_{1} r\right), \\
& v_{l j}=B h_{l}^{(+)}\left(\beta_{1} r\right),
\end{aligned}
$$


where $h_{l}^{(+)}$are spherical Haenkel functions, $\alpha_{1}^{2}=\frac{2 m}{\hbar^{2}}(\lambda+E)$ and $\beta_{1}^{2}=$ $\frac{2 m}{\hbar^{2}}(\lambda-E)$. These solutions correspond to the bound quasiparticle spectrum.

For $E>-\lambda$ the spectrum is continuous and the solutions are:

$$
\begin{aligned}
& u_{l j}=C\left[\cos \left(\delta_{l j}\right) j_{l}\left(\alpha_{1} r\right)-\sin \left(\delta_{l j}\right) n_{l}\left(\alpha_{1} r\right)\right], \\
& v_{l j}=D h_{l}^{(+)}\left(\beta_{1} r\right)
\end{aligned}
$$

where $n_{l}$ are spherical Neumann functions and $\delta_{l j}$ is the phase shift corresponding to the angular momentum $(l j)$.

The constants entering in the wave functions above are fixed by the continuity conditions of the solutions and of their derivatives at $r=a$ and the normalisation. In what follows we discuss only the continuous solutions, i.e., for energies $E>-\lambda$.

Of particular interest are the values of energies for which the wave functions have maximum localisations inside the potential well. These are the regions close to quasiparticle resonances, which can be defined as complex outgoing solutions of HFB equations. In HFB approach one distinguishes two types of quasiparticle resonances. One type corresponds to the single-particle resonances of the mean field. For the pairing correlations an important role is played by those single-particle resonances which are close to the particle thereshold and have relatively high angular momentum.

Another type of quasiparticle resonances corresponds to bound singleparticle states. These resonant states, which appear due to the nondiagonal matrix elements of the pairing field, are specific of the HFB approach. The resonant states corresponding to deep hole states have small widths. The states with very small widths can be eventually treated as quasibound states, normalized to unity in the same volume as the bound quasiparticle states.

A special attention is paid usually to the continuum s1/2 states $[6,7]$. Apart from the deep hole s1/2 states, which in HFB become narrow quasiparticle resonances with high quasiparticle energies, in drip line nuclei one may also find a loosely bound s1/2 single-particle state. In continuum HFB approach this state appears often as a broad quasiparticle resonance (see example below), close to the continuum edge, and its role in pairing correlations cannot be distinguished from the rest of non-resonant s1/2 continuum. This is different from a HF-BCS approach, where the contribution of the loosely bound single-particle s1/2 state to pairing correlations is not mixed with the rest of background single-particle continuum. 
The structure of the continuum discussed above is essentially the same for general, self-consistent HFB calculations. In this case the HFB equations for the continuum spectrum are integrated by starting far from the nucleus with the solution given by Eq.(1.13), which is propagated towards the matching point by the Numerov method. For each quasiparticle energy one calculates, by matching conditions, the phase shift. Then the energies (widths) of quasiparticle resonances are found from the energies where the derivative of the phase shift reaches its maximum (half of its maximum) value. This information is used afterwards to fix an appropriate energy grid (i.e., dense in the energy region of a resonance) for the calculation of the continuum contribution to the particle and pairing densities (see Eqs.(1.9)).

\section{HF-BCS APPROXIMATION}

The HF-BCS approximation is obtained by neglecting in the HFB equations the non-diagonal matrix elements of the pairing field. This means that in the HF-BCS limit one neglects the pairing correlations induced by the pairs formed in states which are not time-reversed partners.

Particularly simple are the HF-BCS equations which include the effect of resonant continuum $[8,9]$ :

$$
\begin{aligned}
\Delta_{i} & =\sum_{j}\langle i, \bar{i}|V| j, \bar{j}\rangle u_{j} v_{j}+\sum_{\nu}\left\langle i, \bar{i}|V| \nu \epsilon_{\nu}, \overline{\nu \epsilon_{\nu}}\right\rangle \int_{I_{\nu}} g_{\nu}^{c}(\epsilon) u_{\nu}(\epsilon) v_{\nu}(\epsilon) d \epsilon \\
\Delta_{\nu}(\epsilon) & =\left(g_{\nu}^{c}(\epsilon) / g_{\nu}(\epsilon)\right)\left(\sum_{j}\langle\nu \epsilon, \overline{\nu \epsilon}|V| j, \bar{j}\rangle u_{j} v_{j}\right. \\
& \left.+\sum_{\nu \prime}\left\langle\nu \epsilon_{\nu}, \overline{\nu \epsilon_{\nu}}|V| \nu \prime \epsilon_{\nu \prime}, \overline{\nu \prime \epsilon_{\nu \prime}}\right\rangle \int_{I_{\nu}} g_{\nu \prime}^{c}(\epsilon \prime) u_{\nu \prime}(\epsilon \prime) v_{\nu \prime}(\epsilon \prime) d \epsilon \prime\right) \\
& \equiv\left(g_{\nu}^{c}(\epsilon) / g_{\nu}(\epsilon)\right) \Delta_{\nu} .
\end{aligned}
$$

where $g_{\nu}^{c}(\epsilon)=\frac{2 j_{\nu}+1}{\pi} \frac{d \delta_{\nu}}{d \epsilon}, g_{\nu}(\epsilon)$ is the total level density and $\delta_{\nu}$ is the phase shift of angular momentum $\left(l_{\nu} j_{\nu}\right)$. In these equations the interaction matrix elements are calculated with the scattering wave functions at resonance energies and normalised inside the volume where the pairing interaction is active. The particle number condition is:

$$
N=\sum_{i} v_{i}^{2}+\sum_{\nu} \int_{I_{\nu}} g_{\nu}^{c}(\epsilon) v_{\nu}^{2}(\epsilon) d \epsilon
$$

The energy factor $g_{\nu}^{c}(\epsilon)$ takes into account the variation of the localisation of scattering states in the energy region of a resonance (i.e., the width effect) and goes to a delta function in the limit of a very narrow width. For more details one can see Ref.[9]. 


\section{APPLICATION: CONTINUUM COUPLING IN DIFFERENT APPROXIMATIONS}

In order to illustrate the approximations discussed above we take as an example the nucleus ${ }^{84} \mathrm{Ni}$, for which HFB calculations with discretized continuum can be found in the literature [4].

Table 1 Energies and widths of quasiparticle resonant states. In the two last columns the total occupancies for each (lj) channel are shown both in full and in box calculations.

\begin{tabular}{|c|c|c|c|c|c|}
\hline $\mathrm{j}$ & 1 & $\mathrm{E}(\mathrm{MeV})$ & $\Gamma(\mathrm{MeV})$ & | Total occ. (full) & | Total occ. (box) \\
\hline $1 / 2$ & 0 & 1.241 & 0.69 & & \\
\hline $1 / 2$ & 0 & 20.956 & 0.154 & & \\
\hline $1 / 2$ & 0 & 43.488 & $10^{-6}$ & & \\
\hline $1 / 2$ & 1 & & & 2.261 & 2.299 \\
\hline $1 / 2$ & 1 & 33.531 & 0.101 & & \\
\hline $3 / 2$ & 1 & 9.806 & 0.60 & 1.993 & 1.994 \\
\hline $3 / 2$ & 1 & 35.058 & 0.104 & 1.999 & 2.016 \\
\hline $3 / 2$ & 2 & 2.354 & 0.641 & & \\
\hline $3 / 2$ & 2 & 22.103 & 0.066 & 1.171 & 1.183 \\
\hline $\begin{array}{l}5 / 2 \\
5 / 2\end{array}$ & $\begin{array}{l}2 \\
2\end{array}$ & $\begin{array}{c}1.816 \\
25.713\end{array}$ & $\begin{array}{l}0.076 \\
0.006\end{array}$ & & \\
\hline $5 / 2$ & 3 & 8.972 & 0.919 & 1.587 & 1.529 \\
\hline $7 / 2$ & 3 & 15.449 & 2.070 & 0.997 & 1.006 \\
\hline & & & & 1.011 & 1.015 \\
\hline $7 / 2$ & 4 & 3.492 & 0.027 & 0.101 & 0.110 \\
\hline $9 / 2$ & 4 & 5.747 & 0.001 & 0050 & 0050 \\
\hline $11 / 2$ & 5 & 5.275 & 0.049 & 0.068 & 0.079 \\
\hline
\end{tabular}

In all calculations discussed below the HF mean field is calculated with the Skyrme interaction SIII. The pairing force is given by Eq.(1.6) and the parameters are the ones used in Ref [4].

First, we present the results given by the continuum HFB equations solved with proper boundary conditions, as defined in the previous sections. These results, referred below as "full" continuum calculations, are compared to the HFB calculations performed with box boundary conditions. 
In Table 1 are shown the quasiparticle resonant states, of hole and particle type. For each $(l j)$ channel are shown also the total occupancy obtained in full and box calculations. One can notice the large widths of quasiparticle states corresponding to bound single-particle states close to the Fermi energy. The widths are obtained from the phaseshift behaviour around $\pi / 2$ except for the three states $s 1 / 2, p 1 / 2$ and $p 3 / 2$ close to the Fermi energy for which the widths are extracted from the occupancy profiles.

The results for the pairing field, pairing density and particle density are shown in Figs.(1-3). On can see that the box calculations generally overestimate the pairing correlations. This is also seen by comparing the Fermi levels (full: $-1.026 \mathrm{MeV}$; box: $-1.205 \mathrm{MeV}$ ), averaged gaps (full: $1.230 \mathrm{MeV}$; box: $1.448 \mathrm{MeV}$ ), pairing energies (full: $-18.092 \mathrm{MeV}$; box: $-24.081 \mathrm{MeV}$ ) and total binding energies (full: $-651.079 \mathrm{MeV}$; box:$654.708 \mathrm{MeV})$.

Next, we compare the results given by the HF-BCS approximation presented in the previous section, to the HFB calculations of Ref.[4]. In Ref. [4] the HFB equations are diagonalised in a basis formed by the single-particle states selected by a box of a finite radius. The singleparticle continuum is cut at $5 \mathrm{MeV}$. This energy cutoff is different from the HFB box calculations presented above, where the energy cutoff is much higher. Up to $5 \mathrm{MeV}$, one finds three single-particle resonances, $d_{3 / 2}, g_{7 / 2}$ and $h_{11 / 2}$, which are treated in the HF-BCS approximation given by Eqs.(1.14). The HFB and HF-BCS results for the pairing field are shown in Fig.4. In the same figure is shown the HF-BCS result obtained by replacing each single-particle resonance by a discrete state, normalized in the same volume as the one used for the bound states. As seen from Fig.4, this result is closer to HFB calculations. This similarity is due to the fact that in the HFB calculations each resonant state is actually represented by a unique state normalised in the box. When the effect of the width of resonant states is included in HF-BCS equations, the pairing correlations are decreasing. This trend is similar with the one observed in continuum HFB calculations presented above. Thus, one can conclude that, in order to take fully into account the effect of resonant continuum upon pairing correlations, one needs to solve the HF-BCS and HFB equations with proper boundary conditions. 


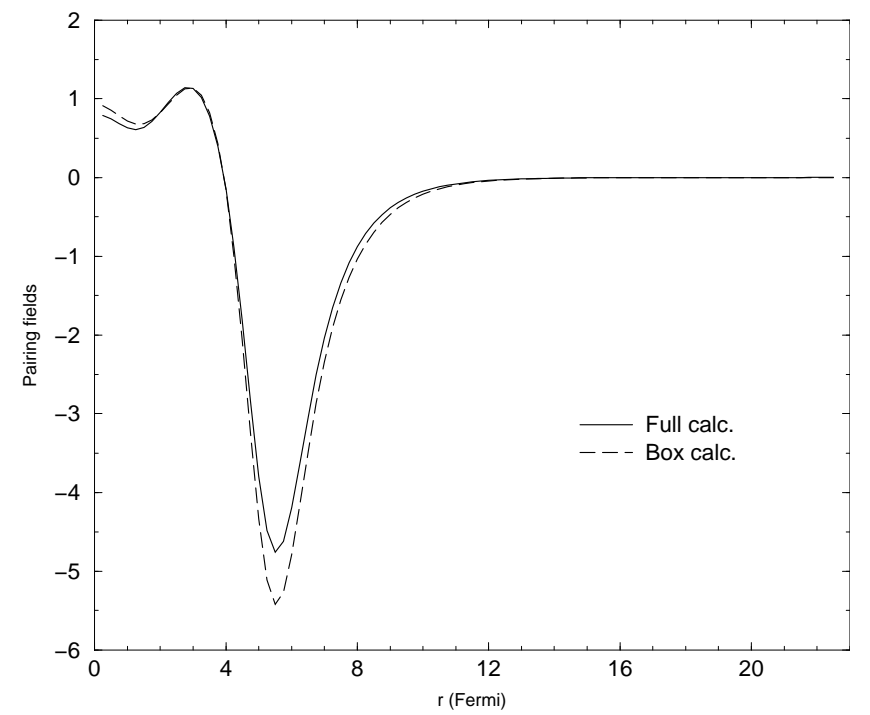

Figure 1 Pairing fields in full (full line) and box (dashed line) calculations.

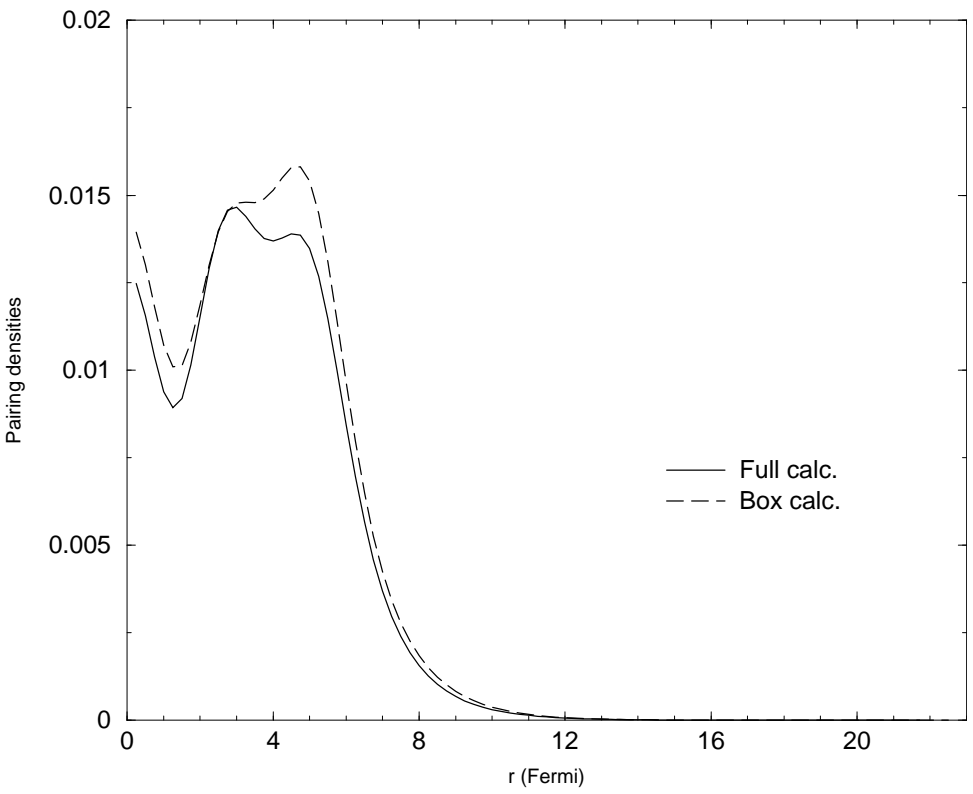

Figure 2 Pairing densities in full (full line) and box (dashed line) calculations. 


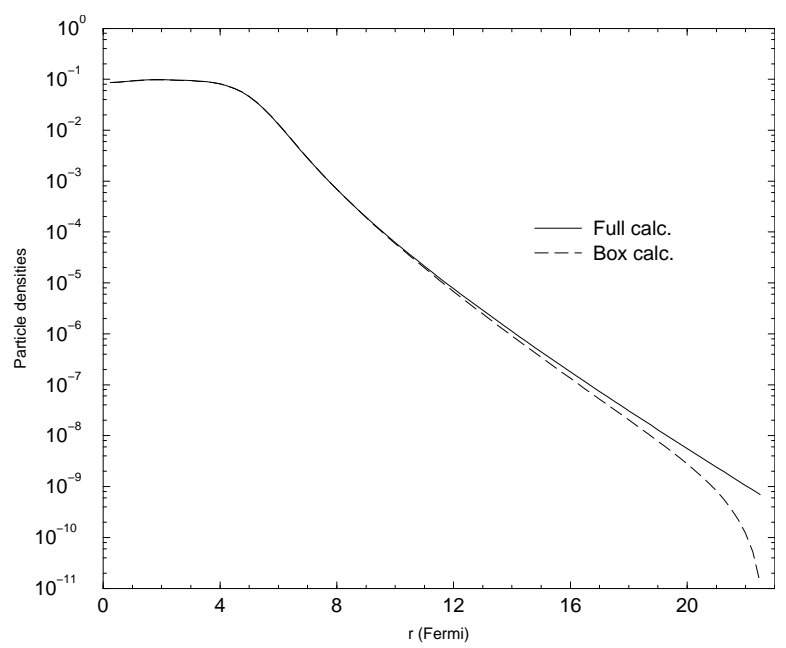

Figure 3 Particle densities in full (full line) and box (dashed line) calculations.

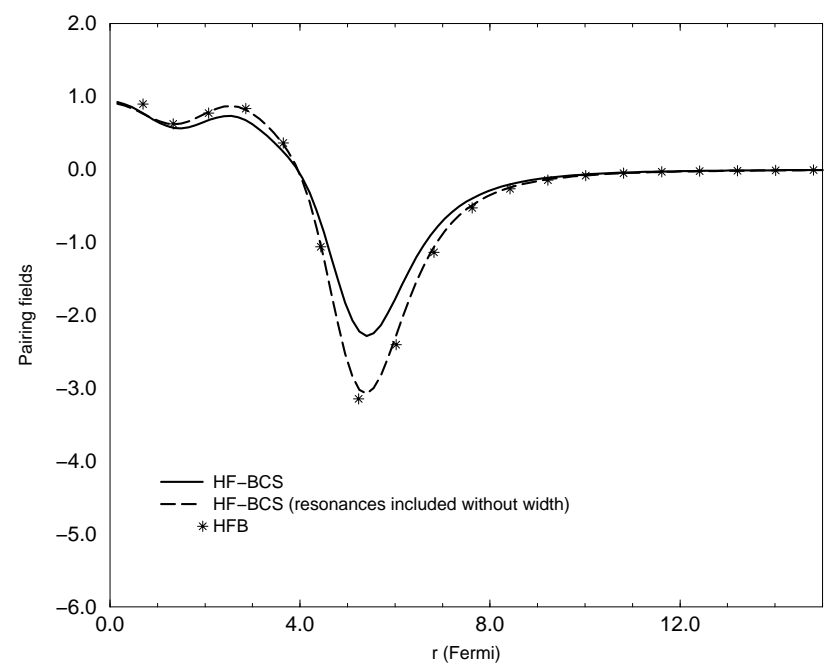

Figure 4 Pairing fields calculated in different approximations: HFB of Ref. [4] (stars), resonant HF-BCS with widths (solid) and without widths (dashed). 


\section{SUMMARY}

In these lectures, we have discussed how the continuum coupling and pairing correlations are calculated in drip line nuclei. The discussion was restricted to the HFB and HF-BCS approximations. In the first part, we have shown how one can construct in the HFB approach solutions with proper boundary conditions for the continuum spectrum. Then, for the case of a simple model, we have analysed the structure of quasiparticle continuum. In particular we have discussed the treatment of resonant continuum both in HFB and HF-BCS approaches.

In the second part we have shown, for the case of a neutron rich nucleus, how different treatments of continuum can affect the pairing correlations. One concludes that the solutions based on discretized continuum (box boundary conditions) can overestimate the pairing correlations when compared with proper continuum HFB or HF-BCS calculations. This may have consequences on the predictions of drip lines.

\section{Acknowledgments}

We thank J. Dobaczewski for providing us the code which solves the HFB equations with box boundary conditions.

\section{References}

[1] P. Ring and P. Schuck, The Nuclear Many-Body Problem (SpringerVerlag, New York, 1990).

[2] J. Dobaczewski, H. Flocard and J. Treiner, Nucl. Phys. A 422, 103 (1984).

[3] J. Dobaczewski, W. Nazarewicz, T.R. Werner, J.F. Berger, C.R. Chinn and J. Dechargé, Phys. Rev. C 53, 2809 (1996).

[4] J. Terasaki, P.-H. Heenen, H. Flocard and P. Bonche, Nucl. Phys. A 600, 371 (1996).

[5] A. Bulgac, preprint nucl-th/9907088.

[6] S.T. Belyaev, A.V. Smirnov, S.V. Tolokonnikov and S.A. Fayans, Sov. J. Nucl. Phys. 45, 783 (1987).

[7] K. Bennaceur, J. Dobaczewski and M. Ploszajjczak, Phys. Rev. C 60, 2809 (1999).

[8] N. Sandulescu, R.J. Liotta and R. Wyss, Phys. Lett. B 394, 6 (1997).

[9] N. Sandulescu, N. Van Giai and R.J. Liotta, Phys. Rev. C 61 (2000) $061301(\mathrm{R})$. 